\title{
Digitalisierung in der ambulanten Gesundheitsversorgung
}

\author{
Tarja Zingg a , Reinhold Sojer ${ }^{b}$, Fabian Röthlisberger ${ }^{c}$

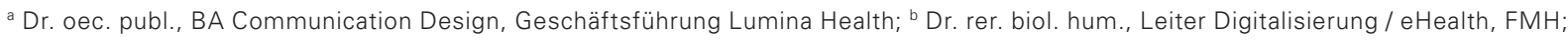 \\ 'Wissenschaftlicher Mitarbeiter Digitalisierung / eHealth, FMH
}

Die Literatur findet sich unter www.saez.ch $\rightarrow$ Aktuelle Ausgabe oder $\rightarrow$ Archiv $\rightarrow 2019 \rightarrow 5$.

\footnotetext{
1 Siehe www.fmh.ch $\rightarrow$ Politik \& Themen $\rightarrow$ eHealth $\rightarrow$ Telemedizin $\rightarrow$ «igitalisierung der ambulanten Gesundheitsversorgung»
}

Die Schweiz hat gute technische und politische Voraussetzungen für die Digitalisierung im ambulanten Gesundheitswesen [1]. Internetzugang und SmartphoneNutzung sind eine Selbstverständlichkeit für die Schweizer Bevölkerung. Digitale Infrastrukturen wie das elektronische Patientendossier (EPD) sind weiter im Aufbau und digitale Anbieter und Angebote im Gesundheitswesen nehmen fortwährend zu. Sowohl Patientinnen und Patienten als auch die Ärzteschaft sind gegenüber der Digitalisierung in der ambulanten Gesundheitsversorgung grundsätzlich und mehrheitlich positiv eingestellt, jedoch führen nur etwa 50\% der Schweizer Praxisärzte eine elektronische Krankengeschichte [2-3].

Um die Digitalisierung im ambulanten Bereich weiter voran zu treiben, sind nun Handlungsschritte erforderlich: Die föderalistisch bedingten und fragmentierten Rechtsgrundlagen müssen harmonisiert, Standards für die Interoperabilität weiter erarbeitet und monetäre und nicht monetäre Anreize geschaffen werden. Damit Patientinnen und Patienten sowie Ärztinnen und Ärzte einen grösstmöglichen Nutzen aus den digitalen Anwendungen ziehen können, müssen sie darüber hinaus über die entsprechenden Kompetenzen verfügen.

Die FMH hat eine Landkarte erarbeitet, die sich mit den Chancen und Risiken der Digitalisierung im ambulanten Gesundheitsbereich auseinandersetzt und ein Leitbild von und für Ärztinnen und Ärzte formuliert. ${ }^{1}$ Nachfolgend werden die bedeutendsten Stärken und Chancen der Digitalisierung im ambulanten Gesundheitsbereich aufgezeigt.

\section{Internetnutzung und -zugang beinahe flächendeckend}

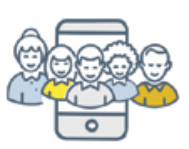

2017 hatten 93\% der Haushalte Zugang zum Internet und rund 80\% der Schweizer Bevölkerung besassen ein Smartphone. Damit sind die technologischen Voraussetzungen für einen breiten, niederschwelligen Zugang zu digitalen Gesundheitsangeboten gegeben [4].

\section{Digitale Gesundheitsangebote sind gefragt}

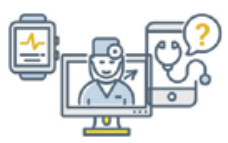

Die Nutzung von und die Nachfrage nach digitalen Angeboten im Gesundheitswesen nehmen stetig zu. So gibt es heute mehr Gesundheits- und Praxiswebseiten denn je. Der Impfausweis ist digital verfügbar und die Möglichkeit, Rezepte via Mail oder Internet anzufordern, werden immer häufiger nachgefragt und angeboten. Eine Mehrheit der Patientinnen und Patienten wünscht sich, mit dem Arzt via Messenger oder E-Mail kommunizieren zu können. Auch mobile Anwendungen wie Health Apps und Wearables sind im Alltag der Bevölkerung zunehmend verankert; 44\% der Schweizer Bevölkerung nutzen Apps für Fitness und Bewegung [3]. Das Angebot an Gesundheits-Apps weltweit in den Kategorien «Gesundheit» und «Fitness \& Medizin» beläuft sich derzeit auf über 300000 [5]. Da wo der Nutzen für Patientinnen und Patienten evident ist, wird die Nachfrage auch weiterhin zunehmen.

\section{Digitale Anbieter und Angebote im Vormarsch:} 2,5 Millionen Patientenkontakte pro Jahr

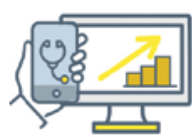
Telemedizinische Gesundheitsberatungen werden heute ortsunabhängig, rund um die Uhr, an 365 Tagen im Jahr bei Fragen zu Prävention, Krankheit, Heilung, Unfall, Mutterschaft oder Linderung von Symptomen von verschiedenen telemedizinischen Organisationen angeboten. Zuweilen reicht eine fundierte telemedizinische Beratung, um mit einer leichten gesundheitlichen Beeinträchtigung richtig umzugehen. So hat die medizinische Online-Beratung inzwischen eine grosse gesellschaftliche Zustimmung erreicht. Heute sind in der Schweiz bereits 13\% der Bevölkerung in einem Telemed-Modell versichert und alleine die vier grössten telemedizinischen Anbieter (Medgate, Medi24, Monvia, Santé24) zusammen verzeichnen rund 2,5 Millionen Patientenkontakte pro Jahr - Tendenz zunehmend [6]. 
Niederschwelliger, breiter Zugang zu Gesundheitswissen

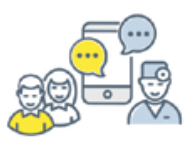

Digitale Angebote wie Gesundheitswebseiten, Telekonsultationen oder Gesundheits-Apps bieten einen niederschwelligen, raum- und zeitunabhängigen Zugang zu Gesundheitsförderung und Prävention. In einem Land, wo 93\% der Haushalte Internetzugang hat [4], führt dies zu einem nahezu flächendeckenden Zugang zu aktuellem Gesundheitswissen. Künftig wird kaum mehr ein Patient einen Arzt aufsuchen, ohne sich vorher im Internet über die Qualität des Arztes und über seine Krankheit informiert zu haben [7]. Allerdings fühlt sich ein Teil der Bevölkerung von der Digitalisierung überfordert: Hier sind besondere Anstrengungen nötig, um deren Kompetenz und Teilnahme zu fördern [8].

Möglichkeiten sind vorhanden, aber noch wenig genutzt

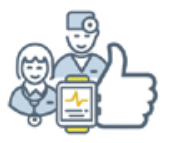

Ärztinnen und Ärzte haben mehrheitlich eine positive Einstellung zu digitalen Gesundheitsangeboten, nutzen deren Möglichkeiten jedoch wenig. Ärztinnen und Ärzte, welche digitale Gesundheitsangebote im beruflichen Alltag integrieren, nutzen insbesondere digitale Angebote zur Verbesserung der Prozesse in der Administration, in der Kommunikation und Information (v.a. in grösseren Praxen), weil der Mehrwert da klar sichtbar ist. Rund die Hälfte der Arztpraxen dokumentieren die Krankheitsgeschichten vollständig digital [3]. Auch bei der Therapieunterstützung setzen sie digitale Angebote zur besseren Diagnose oder individuelleren Behandlung ein (z.B. evidenzbasierte Ressourcen wie uptodate.com). Digitale Anwendungen überzeugen besonders, wenn die Nutzung einfach ist, prozessunterstützend bei Diagnose und Behandlung wirkt, verlässliche Informationen anbietet oder die zeitliche und örtliche Flexibilität erhöht [2].

\section{Telemedizin kann eine gute Sache sein}

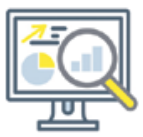

Effizienzsteigerung und Kosteneinsparungen durch die Digitalisierung sind v.a. in der Administration, Information und Kommunikation schnell

sichtbar. Sparpotenzial und Prozessverbesserungen werden aber auch im diagnostisch-therapeutischen Bereich erkannt: Insbesondere bei Behandlungen von gesundheitlichen Störungen, die einfach zu erfassen und schnell lösbar sind oder bei der Therapierung und beim Monitoring gewisser Beschwerden (z.B. COPD) und chronischer Leiden bei denen Patienten zunehmend auch zu Hause betreut werden könnten [9].
Interoperable Infrastruktur von entscheidender Bedeutung

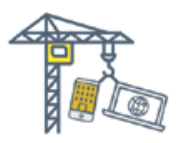

Kompatible, interoperable digitale Infrastrukturen sind das technische Fundament eines modernen Gesundheitswesens. Mit dem weiteren koordinierten Ausbau zukunftsfähiger digitaler Infrastrukturen sinken die Eintrittsbarrieren, Synergien und Effizienzgewinne nehmen zu. Ohne die Vernetzung und Digitalisierung des Gesundheitswesens wird die zeitnahe Umsetzung von neuem medizinischem Wissen in eine verbesserte, patienten- und ergebnisorientierte Versorgung nicht erreichbar sein [10].

\section{Schwächen abbauen, die Risiken minimieren}

Auch wenn mit der eHealth Strategie 2.0 weitere Grundlagen für die Digitalisierung gelegt wurden, sind gerade im ambulanten Gesundheitswesen noch einige Hürden zu überwinden [11]. Schwächen, insbesondere in den Bereichen der Rechtsgrundlagen, des Datenschutzes, der Interoperabilität und der tariflichen Abbildung, sind abzubauen und die Risiken fehlender Wirksamkeitsnachweise und Kompetenzen zu minimieren. So können die teilweise zögerliche Digitalisierung beschleunigt, Ineffizienzen reduziert und Kosten gedämpft werden.

Nachfolgend werden ausgewählte Schwächen und Risiken der Digitalisierung im ambulanten Gesundheitsbereich aufgezeigt.

\section{Datenschutz und Datensicherheit nicht abschliessend geregelt}

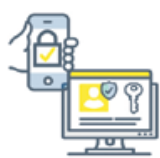
Der Datenschutz und die Datensicherheit sind heikle und emotional besetzte Themen, denn Patientendaten sind besonders schützenswerte Daten. Deshalb unterliegen telemedizinische Leistungen und Anwendungen in erhöhtem Masse dem Datenschutz. Patientendaten sollten nur für die involvierte Ärzteschaft, Therapeuten und Angestellte einsehbar und vor dem Zugriff und der Kenntnisnahme durch Dritte gesichert sein. Insbesondere die sichere Datenübermittlung ist heute noch keine Selbstverständlichkeit. Andererseits birgt die Auswertung grosser und komplexer Datenansammlungen mit gesundheitsrelevanten Informationen immenses Potenzial, um Forschung und Medizin rascher und interdisziplinärer voranzutreiben [12-13].

\section{Tarifliche Abbildung ungenügend}

Noch nicht gelöst sind die Entschädigungsmodelle, die Abrechnung und die Vergütung im Bereich Telemedi- 


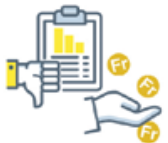

zin sowie der relevanten TARMEDTarifpositionen für verschiedene digitale Leistungsbereiche. Die zurzeit in Frage kommenden Tarifpositionen decken die digitalen Leistungen unvollständig und ungenügend $\mathrm{ab}$. So setzt der TARMED-Tarif heute keinen Anreiz für die Nutzung der Telemedizin, da entsprechende Positionen schlichtweg fehlen. Gerade für kleinere Praxen stellen aber die Investitionskosten bei ungenügender tariflicher Abbildung beziehungsweise fehlender Abgeltungssysteme ein Hindernis für die Integration digitaler Gesundheitsangebote dar [2].

\section{Rechtsgrundlagen heterogen: von Kanton zu Kanton verschieden}

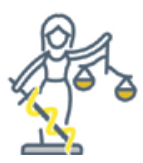

Die Rechtsgrundlagen für die Umsetzung der Digitalisierung in der ambulanten Gesundheitsversorgung sind kantonal sehr unterschiedlich. In vielen Kantonen hinkt der Rechtsrahmen der Realsituation hinterher. So zeigte beispielsweise eine erste Prüfung, dass telemedizinische Leistungen in der Schweiz grundsätzlich wohl möglich sind; allerdings mit kantonalen Unterschieden. In vielen Kantonen (z.B. BL, $\mathrm{ZH})$ ist die ausschliessliche telemedizinische Betreuung von Patienten untersagt oder untersteht ausdrücklich einer Bewilligungspflicht [14].

\section{Realsituation zukunftsorientierter als Rechtsrahmen}

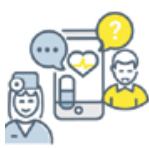

Die Realsituation sieht so aus, dass vor allem die jüngere Ärzteschaft nachfragebedingt digitale Möglichkeiten einsetzt und anbietet [2] - heute mit suboptimalen Rahmenbedingungen, insbesondere bezüglich Abgeltung, Rechtslage und Datenschutz. Eine Mehrheit der Patientinnen und Patienten nutzt die digitalen Möglichkeiten zunehmend in jedem Lebensbereich und fragt digitale Dienstleistungen auch im Gesundheitsbereich nach. Kommunikation und Telekonsultation via Messenger oder E-Mail, elektronische Patientenzuweisung, Gesundheits-Apps und Wearables gehören zu den digitalen Patientenbedürfnissen [3].

\section{Wirksamkeitsanalysen und Evaluations- grundsätze nötig}

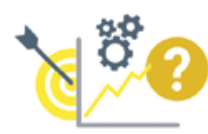

Ambulant tätige Ärztinnen und Ärzte wollen ihre Gesundheitseinrichtung dann digitalisieren, wenn das KostenNutzen-Verhältnis für sie eine positive Bilanz aufweist und der Mehrnutzen für die Patienten aus ihrer Sicht gegeben ist [2]. Wirksamkeits- und Zweckmässigkeitsnachweise digitaler Gesundheitsan- gebote, insbesondere im diagnostisch-therapeutischen Bereich, sind aber noch unzureichend [15]. Angesichts des zunehmenden Kostendrucks wird die Orientierung an der medizinischen Evidenz aber weiter zunehmen. Eine methodisch solide Evaluation von telemedizinisch unterstützten Versorgungsansätzen ist eine wesentliche Voraussetzung für deren breite Umsetzung [16]. Im Sinne der Akzeptanzförderung sollte der administrative vor allem aber diagnostisch-therapeutische Nutzen früh sichtbar werden; denn Effizienzgewinne ermöglichen letztlich, mehr Zeit für die Patienten zu haben.

\section{Zur Digitalisierung befähigen. Im Gleichschritt vorwärts}

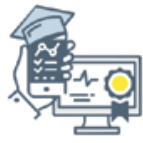

Digitaler Wandel braucht Akzeptanz und gemeinsame Zielvorstellungen. Unterschiedliche digitale Affinitäten und Kompetenzen bei der Ärzteschaft wie bei den Patienten führen zu unterschiedlichem Zugang und unterschiedlicher Nutzung digitaler Angebote. Die Chancen der Digitalisierung werden geschmälert, wenn die digitale Kompetenz der Ärzteschaft und der Patientinnen und Patienten nicht zugleich gefördert wird. Deshalb gehören die Aus- und Weiterbildung und das Change Management zu den wichtigsten strategischen Aufgaben $[8,11,17,18]$.

\section{Zielbild 2022 für die Digitalisierung der ambulanten Gesundheitsversorgung}

Die Digitalisierung im Gesundheitswesen ist ein Megatrend, der oft mit den ganz grossen Themen wie "Blockchain», "Cyber Security" oder "Big Data» in Verbindung gebracht wird. Doch muss die digitale Entwicklung im ambulanten Gesundheitswesen nicht zwingend in derart grossen Dimensionen gedacht werden. Die Möglichkeiten und Auswirkungen der Digitalisierung führten und führen auch in kleineren, pragmatischen Schritten im ambulanten Rahmen zu wichtigen positiven Veränderungen (z. B. in den Bereichen Diabetes- oder Impfmanagement) [19-20]. Handlungsleitend sollen dabei immer die Verbesserung der medizinischen Versorgung sowie mögliche Effizienzgewinne und die Erhöhung der Patientensicherheit sein. Allerdings nützen die Anstrengungen im Kleinen nur, wenn die technischen und politischen Rahmenbedingungen und die Anreize stimmen.

Das Zielbild der Digitalisierung in der ambulanten Gesundheitsversorgung für das Jahr 2022 umfasst sowohl die Erfolgsfaktoren als auch den wünschenswerten zukünftigen Zustand. Für die Entwicklung des Zielbildes waren Wertungen unumgänglich; Wertungen die abhängig von der jeweiligen subjektiven Perspektive 
sind. Deshalb wurden für die Entwicklung dieses Zielbildes die Einschätzungen verschiedener Akteure des Gesundheitswesens eingeholt und so spiegelt das Zielbild 2022 mit seinen zehn Kernaussagen die Sichtweisen aller Akteure gemeinsam wider.

Noch offen ist die Frage der Verantwortlichkeiten für die koordinierte, zeitnahe Formulierung von Mass- nahmen für die Umsetzung der Ziele. Eines ist aber klar: Die erfolgreiche Umsetzung der Digitalisierung im ambulanten Gesundheitswesen gelingt nur unter angemessener Mitwirkung der relevanten Akteure, der Ärzteschaft und der Patientinnen und Patienten in allen Phasen des Vorhabens.

\author{
Digitalisierung der ambulanten Gesundheitsversorgung \\ Zielbild im Öberblick - Ein Leitbild von und für Ärztinnen und Ärzte
}

\title{
\{FMH
}

\section{Zielbild 2022}

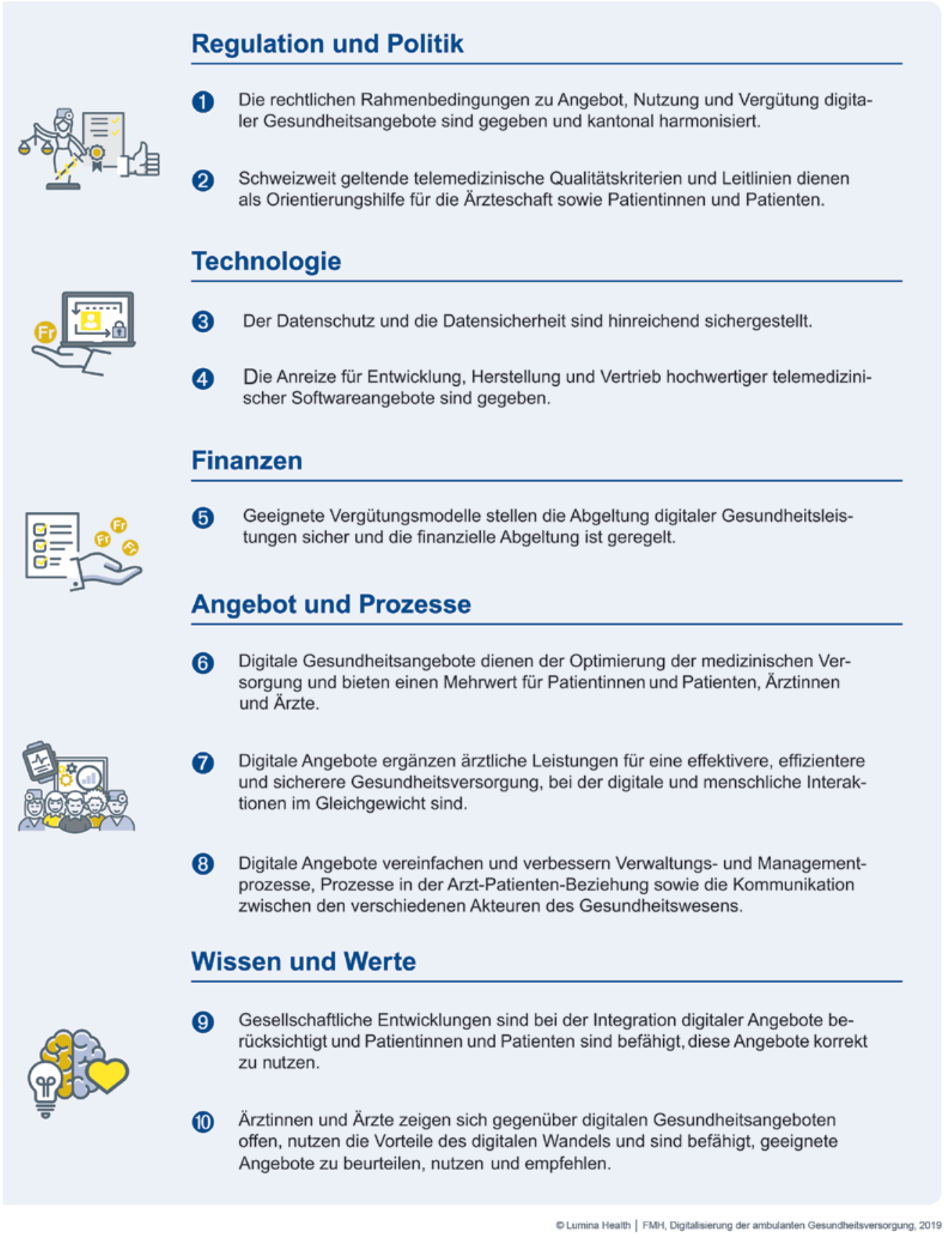




\section{Literatur}

1 C. Meier, Digitale Gesundheit: Schweiz hinkt im internationalen Vergleich hinterher, Medinside, 2018.

2 F. Röthlisberger, R. Sojer, T. Zingg, and O. Rayki, Die Digitalisierung aus Ärztesicht (Teil II), Schweizer Ärztztg., 2018;99(48):1686-9.

3 Golder L, Jans C, Swiss eHealth Barometer 2018, 2018.

4 Bundesamt für Statistik, Internetzugang der Haushalte und Internetnutzung der Bevölkerung in der Schweiz, 2017.

5 Research2Guidance, mHealth App Economics 2017: Current Status and Future Trends in Mobile Health, research2guidance Berlin, Germany, 2017.

6 C. Von Gossler and C. Klauser, Telemedizin in der Schweiz - das Beispiel Medgate, Deutsche Medizinische Wochenschrift, 2017.

7 N. Van Riel, K. Auwerx, P. Debbaut, S. Van Hees, and B. Schoenmakers, The effect of Dr Google on doctor-patient encounters in primary care: a quantitative, observational, cross-sectional study, BJGP Open, 2017.

8 H. Kim and B. Xie, Health literacy in the eHealth era: A systematic review of the literature, Patient Education and Counseling, 2017.

9 T. L. Michaud, J. Zhou, M. A. McCarthy, M. Siahpush, and D. Su, Costs of home-based telemedicine programs: A systematic review, Int. J. Technol. Assess. Health Care, vol. 34, no. 4, pp. 410-418, Jan. 2018.

10 T. Kostera and T. Thranberend. Von anderen Ländern lernen: Wie gelingt die Digitalisierung des Gesundheitswesens? Internationale Vergleichsstudie gestartet, 2018.

11 eHealth Suisse, Strategie eHealth Schweiz 2.0 2018-2022, 2018.

12 N. Mehta and A. Pandit, Concurrence of big data analytics and healthcare: A systematic review, International Journal of Medical Informatics, 2018.

13 C. S. Kruse, R. Goswamy, Y. Raval, and S. Marawi, Challenges and Opportunities of Big Data in Health Care: A Systematic Review, IMIR Med. Informatics, 2016.

14 Koordinationsorgan eHealth Bund-Kantone, eHealth Suisse: mobile Health (mHealth) Empfehlungen I, 2017.

15 C. Sanyal, P. Stolee, D. Juzwishin, and D. Husereau, Economic evaluations of eHealth technologies: A systematic review, PLoS ONE, 2018.

16 A. Enam, J. Torres-Bonilla, and H. Eriksson, Evidence-Based Evaluation of eHealth Interventions: Systematic Literature Review, J. Med. Internet Res., 2018.

17 L. F. M. Ariens, et al., Barriers and facilitators to ehealth use in daily practice: Perspectives of patients and professionals in dermatology, J. Med. Internet Res., 2017.

18 C. Scott Kruse, P. Karem, K. Shifflett, L. Vegi, K. Ravi, and M. Brooks, Evaluating barriers to adopting telemedicine worldwide: A systematic review, Journal of Telemedicine and Telecare, 2018.

19 M. Fadda, E. Galimberti, M. Fiordelli, L. Romanò, A. Zanetti, and P. J. Schulz, Effectiveness of a smartphone app to increase parents' knowledge and empowerment in the MMR vaccination decision: A randomized controlled trial, Hum. Vaccines Immunother., 2017.

20 S. Kitsiou, G. Paré, M. Jaana, and B. Gerber, Effectiveness of mHealth interventions for patients with diabetes: An overview of systematic reviews, PLoS ONE, 2017 